\title{
Inhomogeneous broadening of phosphorus donor lines in the far-infrared spectra of single-crystalline SiGe
}

\author{
S. A. Lynch* and G. Matmon \\ London Centre for Nanotechnology, University College London, 17-19 Gordon Street, London WC1H 0AH, United Kingdom
}

S. G. Pavlov

Institute of Planetary Research, German Aerospace Center (DLR), Rutherfordstr. 2, 12489 Berlin, Germany

K. L. Litvinenko

Advanced Technology Institute, University of Surrey, Guildford GU2 7XH, United Kingdom

B. Redlich and A. F. G. van der Meer

FOM Institute for Plasma Physics Rijnhuizen, P.O. Box 1207, NL-3430 BE Nieuwegein, The Netherlands

N. V. Abrosimov

Leibniz Institute of Crystal Growth, Max-Born-Str. 2, 12489 Berlin, Germany

H.-W. Hübers ${ }^{\dagger}$

Institut für Optik und Atomare Physik, Technische Universität Berlin, Hardenbergstraße 36, 10623 Berlin, Germany

(Received 15 November 2010; published 14 December 2010)

\begin{abstract}
The origins of line broadening in the far-infrared spectrum of phosphorus donors in SiGe are investigated. Using a combination of Fourier transform infrared (FT-IR) spectroscopy and time-resolved pump-probe measurements, we show that the line shapes are dominated by inhomogenous broadening. Experimental FT-IR absorbance spectra measured in the temperature range $6-150 \mathrm{~K}$ are presented for three different Ge contents. Additional spectra of pure phosphorus doped silicon recorded under similar experimental conditions are presented and compared with the SiGe results. We propose a simple quantitative model to simulate the line broadening in our experimental spectra. Our model takes into account the compositional variations in the random SiGe binary alloy and its effect on the permittivity of the environment around each donor. We also show that the addition of small amounts Ge to Si single crystals has little detrimental effect on the lifetime of the excited infrared electronic energy levels, despite the observed line broadening.
\end{abstract}

DOI: 10.1103/PhysRevB.82.245206

PACS number(s): 78.30.Am, 61.72.uf, 71.55.-i, 78.47.D-

\section{INTRODUCTION}

In the last decade, there has been resurgent interest in the physics of group- $\mathrm{V}$ donors in silicon, as evidenced by a dramatic increase of activity in this area. Much of this renewed interest stemmed from a proposal by Kane that the material system might be exploited to realize a quantum computer. ${ }^{1}$ Many experiments have recently been performed to investigate the feasibility of this scheme. Pulsed electron paramagnetic resonance experiments have revealed extraordinarily long donor spin lifetimes, of order $60 \mathrm{~ms}$ at $7 \mathrm{~K}$ for ${ }^{28} \mathrm{Si}: \mathrm{P}$ single-isotope material, ${ }^{2}$ and spin readout schemes have been proposed. ${ }^{3}$ High-resolution Fourier transform infrared (FTIR) spectra in similar ${ }^{28} \mathrm{Si}: \mathrm{P}$ material has also revealed some of the narrowest spectral lines ever measured in the solid state. ${ }^{4}$ Complementary time domain terahertz (THz) pumpprobe experiments show that the lifetime of the excited Si:P Rydberg states is of order 200 ps (Ref. 5) (in excellent agreement with the best frequency domain ${ }^{28} \mathrm{Si}: \mathrm{P}$ measurements). Coherent control of $\mathrm{Si}: \mathrm{P}$ states with $\mathrm{THz}$ optical pulses has also just been demonstrated. ${ }^{6}$ A second important driver for this research has been the invention of the optically pumped silicon $\mathrm{THz}$ laser ${ }^{7}$ and lasing has now been demonstrated for all four group-V donors. ${ }^{8-10}$ In addition to this, the micro- electronics industry has now moved to SiGe virtual substrates and strained SiGe epitaxial layers in its search for faster on-chip components. ${ }^{11}$ It is therefore important to understand exactly how shallow impurities such as the group-V donors behave in this technologically important material system.

\section{EXPERIMENTAL}

\section{A. Sample growth}

For the purposes of these experiments, three single crystals of $\mathrm{Si}_{1-x} \mathrm{Ge}_{x}$ orientated along the $\langle 110\rangle$ axis with diameter $40 \mathrm{~mm}$, and with Ge content, $0.9 \%, 3 \%$, and $6 \%$, were grown in an argon atmosphere using the Czochralski technique. A detailed description of the growth process can be found here. $^{12}$ The resistivity and hence phosphorus donor concentration was kept approximately constant. A phosphorus doped pure silicon sample of comparable resistivity was also chosen as a reference. The sample details are summarized in Table I. Square samples with dimensions $7 \mathrm{~mm} \times 7 \mathrm{~mm}$ and $1 \mathrm{~mm}$ thick were then cut from the single crystal. There were no subsequent additional polishing steps; the samples were studied as cut. 
TABLE I. Sample name, Ge content, resistivity, and phosphorus concentration.

\begin{tabular}{llcc}
\hline \hline Sample name & $\begin{array}{c}\text { Ge content } \\
(\%)\end{array}$ & $\begin{array}{c}\text { Resistivity } \\
(\Omega \mathrm{cm})\end{array}$ & $\begin{array}{c}\text { Conc. } \\
\left(\mathrm{cm}^{-3}\right)\end{array}$ \\
\hline Si:P V2682 & 0 & 1.2 & $4 \times 10^{15}$ \\
SiGe:P 236 & 0.9 & 1.0 & $5 \times 10^{15}$ \\
SiGe:P 222 & 3.0 & 2.0 & $2.3 \times 10^{15}$ \\
SiGe:P 223 & 6.0 & 2.0 & $2.3 \times 10^{15}$ \\
\hline \hline
\end{tabular}

\section{B. Far-infrared spectroscopy}

FT-IR absorption spectroscopy was performed using our Bruker 125 HR FT-IR vacuum spectrometer. The samples were individually mounted on a custom oxygen-free copper mount, which was in turn attached to the cold finger of an Oxford Instruments flow cryostat. The sample mount was carefully designed so that the samples were not subjected to any strain while still retaining good thermal contact with the cold finger. This is a key point: in our previous experimental studies, significant strain effects were observed in the absorption spectra of glued silicon samples on cooling to liquid helium temperatures. The full beam path was under vacuum. Figure 1 shows absorbance spectra for the three $\mathrm{Si}_{1-x} \mathrm{Ge}_{x}: \mathrm{P}$ samples and the Si:P reference for temperatures between 6 and $150 \mathrm{~K}$. The top plot shows the familiar shallow impurity states and their temperature dependence. At this donor concentration and optical thickness the $1 s\left(A_{1}\right)$ to $2 p_{ \pm}$and $1 s\left(A_{1}\right)$ to $3 p_{ \pm}$lines are saturated. As the sample temperature rises, the two higher lying valley orbit split-off ground states $1 s(E)$ and $1 s\left(T_{2}\right)$ become thermally populated, leading to additional absorption lines in the $20-30 \mathrm{meV}$ region. ${ }^{13}$ These additional lines can be seen when the sample temperature reaches about $40 \mathrm{~K}$, and they become particularly strong, in the temperature range $60-90 \mathrm{~K}$. The strongest absorption lines seen here are from $1 s(E)$ and $1 s\left(T_{2}\right)$ valley orbit ground states to the $2 p_{0}$ and $2 p_{ \pm}$states. Transitions to the higher lying $p$ states are not normally observed. This is because the higher $p$ states are much closer to the continuum edge and thus more easily ionized at elevated temperatures. The absorption cross section for transitions to higher $p$ states is also smaller. With the addition of a small amount of $\mathrm{Ge}$ to the $\mathrm{Si}$ matrix the phosphorus donor lines are no longer sharp. At 6 $\mathrm{K}$ the $0.9 \% \mathrm{Si}_{1-x} \mathrm{Ge}_{x}$ sample shows a strong broad spectral feature close to where the $2 p_{ \pm}$line would be in pure Si:P. At higher temperatures a broad feature which contains contributions from $1 s(E)$ and $1 s\left(T_{2}\right)$ to $2 p_{+}$transitions, can be observed around $20-30 \mathrm{meV}$. The spectral features become increasingly broader as the Ge content is increased. These results agree well with photoconductivity measurements described in Ref. 14.

\section{Time-resolved spectroscopy}

For the time-resolved pump-probe spectroscopy measurements we concentrated on the $0.9 \% \mathrm{Si}_{1-x} \mathrm{Ge}_{x}$ sample because it still had discernible spectral peaks. In particular we investigated the behavior of the $T_{1}$ lifetime of the spectral feature
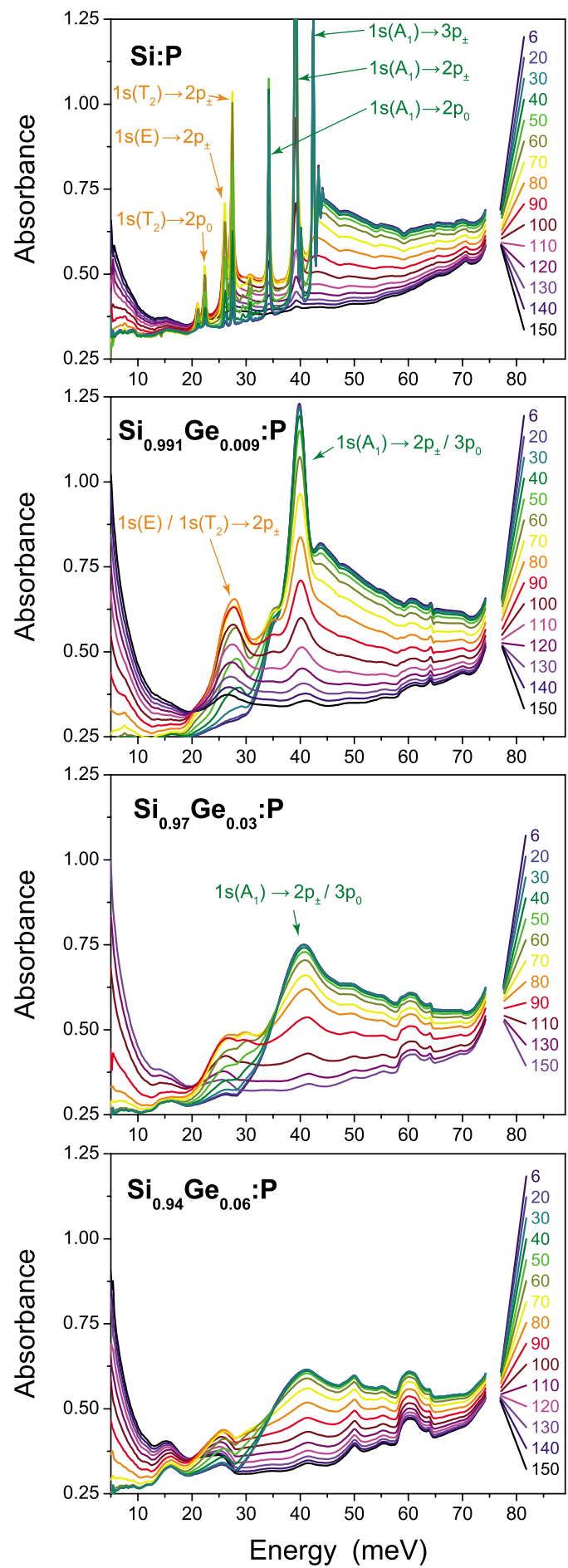

FIG. 1. (Color online) Far-infrared absorbance spectra for Si:P and samples of $\mathrm{Si}_{1-x} \mathrm{Ge}_{x}$ with different Ge contents. The curves are color coded to show different sample temperatures. Transitions from the ground state $1 s\left(A_{1}\right)$ to excited $p$ states (green online), and transitions from higher lying valley orbit split-off ground states $1 s(E)$ and $1 s\left(T_{2}\right)$ to the excited $p$ states (orange online) are labeled.

corresponding to the broadened $1 s\left(A_{1}\right)$ to $2 p_{ \pm}$line at 38.1 $\mathrm{meV}(32.5 \mu \mathrm{m})$. Here we define $T_{1}$ according to the simple exponential decay function, $y_{0}+C_{0} \exp \left(-t / T_{1}\right)$, and we fit this function to the data. Figure 2 shows a diagram of the experimental setup. We used the free electron laser at the 


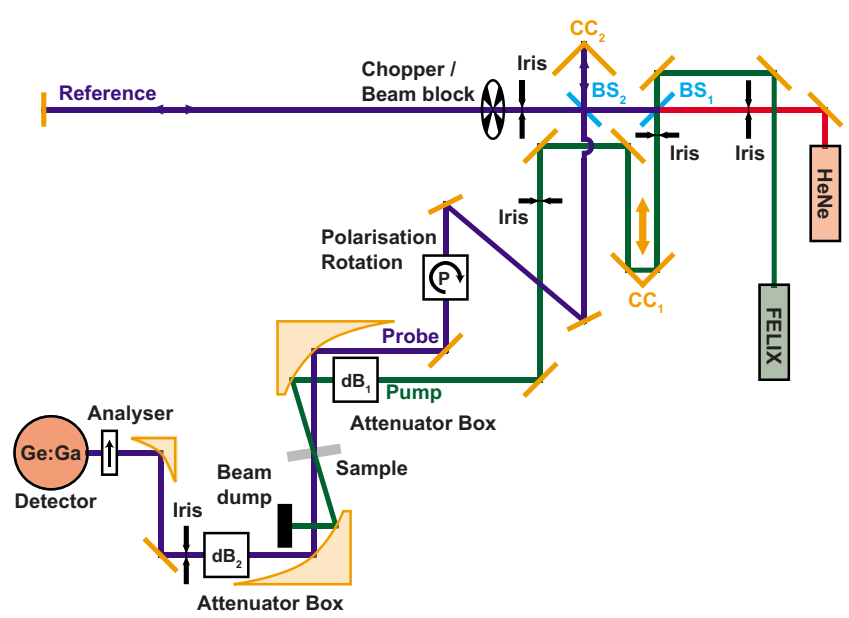

FIG. 2. (Color online) Schematic showing the layout of the pump-probe experimental setup at FELIX.

FOM Institute in the Netherlands (FELIX) as a source of short optical $(\mathrm{THz})$ pulses. The beam path of the stronger pump pulse is labeled "pump" (green online) and the weaker probe pulse is labeled "probe" (blue online). The pump traverses an optical delay line, allowing the relative delay between the pump and probe to be controlled. The polarization of the probe is rotated by $90^{\circ}$ in order to discriminate it from the pump at the detector. A portion of the probe beam (labeled reference) also passes through a much longer optical delay $(\approx 20 \mathrm{~ns})$. All beam paths were in a dry nitrogen atmosphere. The recorded probe signal is derived from the difference between the directly measured probe and reference signal, resulting in a background free signal. As a first experiment we investigated how the exponential decay of the probe transmission varied with the pump wavelength and these results are shown on the top half of Fig. 3. The $T_{1}$ lifetime was smallest, showing that the decay was fastest, on the high-energy side of the broadened $1 s\left(A_{1}\right)$ to $2 p_{ \pm}$peak. This is because of significant recombination transitions into the other nearby higher lying Rydberg donor levels (such as the $3 p_{0}$ state) which give rise to other parallel decay paths. On the low-energy side, however, the lifetime remains reasonably constant until near the energy of where the $1 s\left(A_{1}\right)$ to $2 p_{0}$ line would be observed in the spectrum of pure Si:P. Here, the value of the $T_{1}$ lifetime grows larger again, and begins to approach the previously published lifetime of the unperturbed $2 p_{0}$ state in pure $\mathrm{Si}: \mathrm{P}(205 \mathrm{ps}) .{ }^{5}$ The pump-probe signal became weaker below $34 \mathrm{meV}$ and completely extinguished at $29 \mathrm{meV}$ (not shown). We chose a pump wavelength on the lower energy side of the broadened $1 s\left(A_{1}\right)$ to $2 p_{ \pm}$peak to minimise contributions from the higher lying Rydberg states. We then verified that the pump power did not significantly affect the exponential decay of the probe transmission. The results are shown in Fig. 3 and demonstrate that the variation of measured $T_{1}$ values is at most $11.5 \%$ from the mean value of $118 \mathrm{ps}$ over three orders of magnitude of incident pump pulse power.

Figure 4 shows how the lifetime varies as a function of temperature. The $T_{1}$ decay constants as a function of temperature are also plotted above. $T_{1}$ is longest at lowest temperatures (115-118 ps) and decreases as the sample tempera-

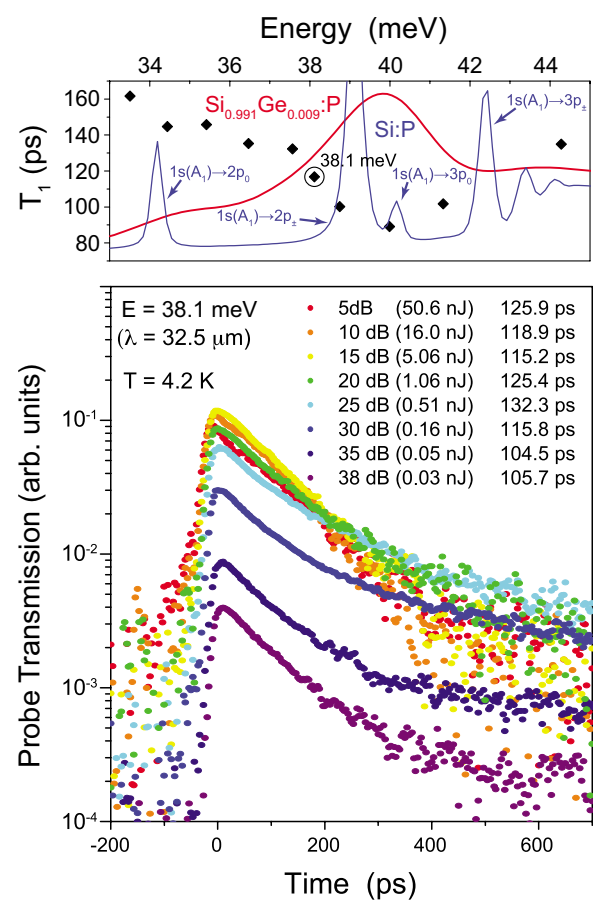

FIG. 3. (Color online) Top: the solid diamonds show the $T_{1}$ decay constants plotted against the pump photon energy (frequency). The solid lines (blue and red) are the FTIR absorbance spectra for $\mathrm{Si}: \mathrm{P}$ and $\mathrm{Si}_{0.991} \mathrm{Ge}_{0.009}$ recorded at $6 \mathrm{~K}$. Bottom: Recovery of the probe absorption as function of time delay from the pump pulse for several different pump attenuations. The $T_{1}$ decay constants for these curves are tabulated against FELIX attenuation and (pulse energy) in the top right corner of this panel.

ture rises. Above $110 \mathrm{~K}$ it was no longer possible to obtain an accurate determination of $T_{1}$ because the decay is shorter than the length of the laser pump pulse. The decrease in $T_{1}$ lifetime can be explained by stimulated phonon emission. It is known that the lifetime of the excited donor states in $\mathrm{Si}: \mathrm{P}$ is mediated by phonon emission rather than photon emission. ${ }^{5}$ Warming the sample increases the population of the phonon bath, which in turn increases the probability of stimulating phonon emission. This is analogous to how an intense optical laser field can stimulate the emission of a photon.

The most important point, however, is that while the $T_{1}$ decay constant of the $2 p_{ \pm}$state changes by an order of magnitude (from $115 \mathrm{ps}$ to $<10 \mathrm{ps}$ ) in the temperature range $6-130 \mathrm{~K}$, there is no corresponding change in the linewidth of the associated spectral feature over the same temperature range (see Fig. 1). These experimental results provide direct evidence that the line broadening is not lifetime related.

\section{MODELING THE LINE BROADENING}

We have derived a simple model to describe the observed line broadening based loosely on the approach described by Schubert et al., ${ }^{15}$ in their paper on alloy broadening in the III-V ternary material system $\mathrm{Al}_{x} \mathrm{Ga}_{1-x} \mathrm{As}$. Since the $\mathrm{Si}_{1-x} \mathrm{Ge}_{x}$ material used for this work is a random binary alloy, the probability of finding $z$ Ge atoms within a prescribed vol- 


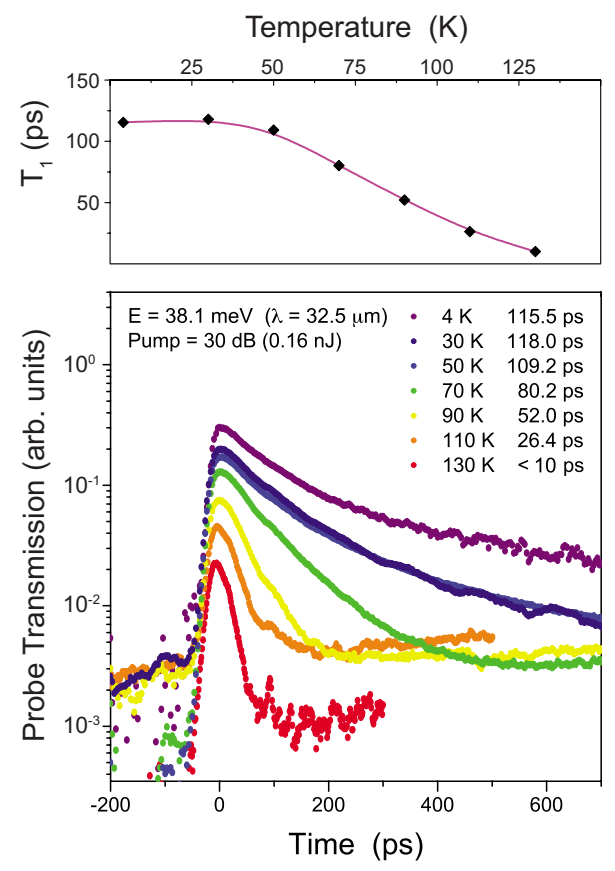

FIG. 4. (Color online) Top: the solid diamonds are the $T_{1}$ lifetime decay constants plotted as a function of temperature. The connecting line (purple) is merely a guide to the eye. Bottom: recovery of the probe absorption as function with time delay from the pump pulse for several sample temperatures. The $T_{1}$ decay constants for these curves are tabulated against temperature in the top right corner of this panel.

ume, $V$, can thus be described by the binomial distribution, where $\rho$ is the density of lattice sites.

$$
p(z)=\left(\begin{array}{c}
\rho V \\
z
\end{array}\right) x^{z}(1-x)^{\rho V-z} .
$$

For the case where the number of trials is large (in this case $\rho V)$, the distribution is Gaussian, with mean value $\mu=\rho V x$, and variance $\sigma^{2}=\rho V x(1-x)$. Since the density of silicon lattice sites, $\rho=4.993 \times 10^{22} \mathrm{~cm}^{-3}$, and the radius of the $1 s\left(A_{1}\right)$ phosphorus donor Rydberg orbital is of order $r=3 \mathrm{~nm}$, even the lowest lying donor orbital encompasses of the order of 10000 lattice sites. The Gaussian approximation for the distribution of $\mathrm{Ge}$ atoms is therefore valid. We know the mean value of the distribution precisely, since it is just the average content, $x$, of $\mathrm{Ge}$ atoms in the random binary alloy so that the variance simplifies to $\sigma_{x}^{2}=x(1-x)$. We will now use this relation to calculate the infrared energy distribution of the phosphorus donors.

The energy of the phosphorus donors can be described by the modified Bohr formula, where $m_{0}$ is the electron mass, $m^{*}$ is the reduced effective mass, $\boldsymbol{\epsilon}_{r}$ is the relative dielectric permittivity, $R_{H}$ is Rydberg constant for hydrogen, and $N$ is the principle quantum number. ${ }^{16}$

$$
E=\frac{m^{*}}{m_{0}} \frac{1}{\epsilon_{r}^{2}} R_{H}\left(1-\frac{1}{N^{2}}\right) .
$$

According to this formula, the energy of the donor depends on both the effective mass, $m^{*}$, and the relative dielectric
TABLE II. Ge Content, Ge distribution width $\sigma_{x}$, and width of the phosphorus donor energy distribution, $\sigma_{E}$, used to calculate Fig. 5 using Eq. (6).

\begin{tabular}{ccc}
\hline \hline $\begin{array}{c}\text { Ge content } \\
(\%)\end{array}$ & $\sigma_{x}$ & $\begin{array}{c}\sigma_{E} \\
(\mathrm{meV})\end{array}$ \\
\hline 0.9 & 0.0949 & 1.607 \\
3.0 & 0.1732 & 2.927 \\
6.0 & 0.2449 & 4.127 \\
\hline \hline
\end{tabular}

permittivity $\epsilon_{r}$. For small Ge fractions, the lattice parameter of the $\mathrm{Si}_{1-x} \mathrm{Ge}_{x}$ unit cell varies by less than $0.4 \%$ for $\mathrm{Ge}$ contents up to $x=0.1$ so we choose to treat $m^{*}$ as constant. ${ }^{17}$ There is a significant change, however, in the value of $\epsilon_{r}$ over the same range. We can calculate the variation of $\epsilon_{r}$ as a function of the Ge content by using the relation $n=\sqrt{\epsilon \mu}$ and the expression derived by Humlícek et al. ${ }^{18}$ for the change in far-infrared refractive index of $\mathrm{Si}_{1-x} \mathrm{Ge}_{x}$ as a function of Ge content.

$$
n(x)=4.01-0.81(1-x)+0.22(1-x)^{2} .
$$

For small $x$ we calculate the dependence of $\epsilon_{r}$ on $x$.

$$
\epsilon_{r}(x)=11.7+2.53 x+0.14 x^{2}
$$

Differentiating the modified Bohr formula [Eq. (2)] with respect to $x$ we get the following expression:

$$
\frac{d E}{d x}=-\frac{2 E}{\epsilon_{r}} \frac{d \epsilon_{r}}{d x} .
$$

Substituting for $\epsilon_{r}$ and $d \epsilon_{r} / d x$, and ignoring higher powers of $x$, we can calculate the change in phosphorus donor energies, $\Delta E$, in terms of the change in Ge fraction, $\Delta x$.

$$
\Delta E=\frac{2(2.53+0.28 x)}{11.7+2.53 x} E \Delta x .
$$

Thus, from knowing the variance of the distribution of $\mathrm{Ge}$ donors, $\sigma^{2}$, we can calculate variance of phosphorus donor energies as a function of Ge content. We now use this formula to calculate the width of the energy distribution for the most prominent line in the far-infrared absorption spectrum of $\mathrm{Si}: \mathrm{P}$, which has a peak absorption centered at $E=39.2 \mathrm{meV}$. Thus, we see the line is inhomogenously broadened due to the random distribution of the binary $\mathrm{Si}_{1-x} \mathrm{Ge}_{x}$ alloy, and the calculated width of this energy distribution, $\sigma_{E}$, for our three $\mathrm{Si}_{1-x} \mathrm{Ge}_{x}$ samples is given in Table II.

In order to simulate a line broadened spectrum, we take the pure experimental $\mathrm{Si}: \mathrm{P}$ spectrum, in the form of a data vector $g\left(x_{k}\right)$ and convolve it with a Gaussian energy distribution $f\left(x_{k}\right)$. Here $k$ is a running index $(1 \rightarrow M)$, and the Gaussian energy distribution is defined by Eq. (7) with mean value $\mu$ and variance $\sigma_{E}^{2}$. 


$$
f\left(x_{k}\right)=\frac{1}{\sigma \sqrt{2 \pi}} \exp \left[-\frac{1}{2}\left(\frac{x_{k}-\mu}{\sigma_{E}}\right)^{2}\right] .
$$

We define $h\left(x_{k}\right)$, the simulated $\mathrm{Si}_{1-x} \mathrm{Ge}_{x}$ spectrum, as the convolution of $f\left(x_{k}\right)$ and $g\left(x_{k}\right)$.

$$
h\left(x_{k}\right)=(f * g)\left(x_{k}\right) .
$$

In order to calculate this convolution, we take the discrete Fourier transform of both $f\left(x_{k}\right)$ and $g\left(x_{k}\right)$ according to the formula

$$
\hat{f}\left(x_{j}\right)=\sum_{j=1}^{M} f\left(x_{j}\right) \omega_{M}^{(j-1)(k-1)},
$$

where $\omega_{M}=\exp (-2 \pi i / M)$ and $j$ is another running index $(1 \rightarrow M)$. We then calculate the product

$$
\hat{h}\left(x_{k}\right)=\hat{f}\left(x_{k}\right) \hat{g}\left(x_{k}\right)
$$

and finally recover the simulated line broadened spectrum of phosphorus in $\mathrm{Si}_{1-x} \mathrm{Ge}_{x}$, by taking the inverse discrete Fourier transform

$$
h\left(x_{k}\right)=\frac{1}{M} \sum_{k=1}^{M} \hat{h}\left(x_{k}\right) \omega_{M}^{-(j-1)(k-1)}
$$

again with the same definition of $\omega_{M}$.

An example of this calculation is shown in Fig. 5. The solid black line shows the experimental absorbance spectrum recorded at $6 \mathrm{~K}$ for the three $\mathrm{Si}_{1-x} \mathrm{Ge}_{x}$ samples described in Table I. The dashed black line shows the simulated line broadened spectrum which was calculated from the $6 \mathrm{~K}$ absorbance spectrum of pure Si:P. The simulated spectra show both excellent qualitative and quantitative agreement with the experimental spectra. The $0.9 \%$ simulated spectrum differs mainly in the height of the continuum absorbance edge. On the low energy side of the main absorption peak, the shoulder [which is the remnant of the $1 s\left(A_{1}\right)$ to $2 p_{0}$ line] agrees well. The minor disagreement in continuum absorbance levels is easily explained because the resistivity of this particular sample was different from both the other $\mathrm{Si}_{1-x} \mathrm{Ge}_{x}$ samples (3\% and 6\%), and the pure $\mathrm{Si}: \mathrm{P}$ sample chosen to simulate the broadening. There is good qualitative agreement in the line shapes although it does appear that our simulation over estimates the broadening slightly.

Kräussmann et al. ${ }^{19}$ studied the activation energy of phosphorus donors in silicon-rich polycrystalline SiGe. They concluded that the linewidth could be affected by two possible mechanisms: through the local value of the dielectric constant or through an average strain produced by the volume misfit of the Ge atoms. They estimated that the dielectric shift would result in a line broadening $\sigma_{E}=\sqrt{x} \times 1.3 \mathrm{meV}$, and the strain shift would result in a broadening of $\sigma_{E}=\sqrt{x}$ $\times 0.826 \mathrm{meV}$, both of which were an order of magnitude too small to match their experimentally observed broadening. They therefore concluded that the actual density fluctuations of $\mathrm{Ge}$ in their polycrystalline samples were much higher than could reasonably be explained by a random distribution. Comparing our experimental results with those from our simulation, however, shows that it is sufficient to take into
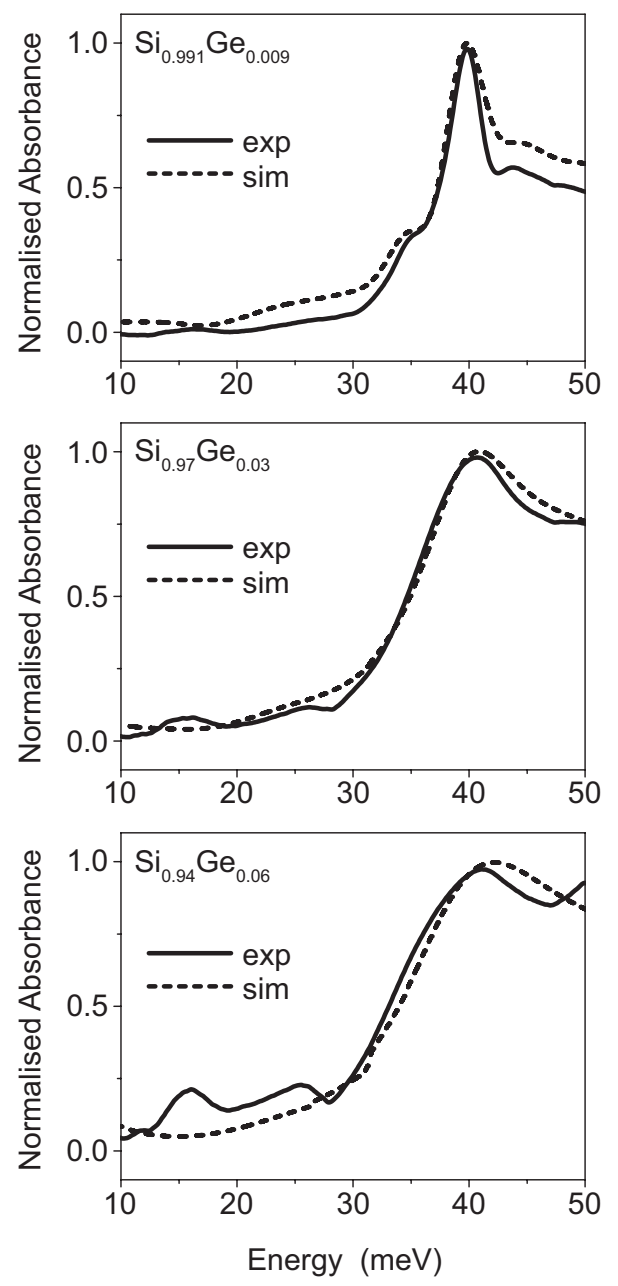

FIG. 5. Normalized $\mathrm{Si}_{1-x} \mathrm{Ge}_{x}$ absorbance spectra at $6 \mathrm{~K}$ for three different Ge contents. The solid line is the experimental spectrum and the dashed line is the simulated spectrum. Top: $0.9 \% \mathrm{Ge}$. Middle: $3 \%$ Ge. Bottom: $6 \%$ Ge.

account the local variation of permittivity in order to fully describe the line broadening. The simulation also provides further additional evidence that the line broadening in single crystal $\mathrm{SiGe}$ is inhomogenous.

\section{DISCUSSION}

We now discuss our findings and their implications. Our results are important for the design of $\mathrm{THz} \mathrm{SiGe}$ quantum cascade laser active regions. These designs have previously avoided the 30-40 meV region, where the absorption lines arising from the shallow acceptor states of boron in $\mathrm{Si}$ (Si:B) are concentrated since they would lead to increased cavity losses. ${ }^{20,21}$ Our results for SiGe:P show that shallow donor and acceptor impurities are likely to give rise to cavity losses across a much wider $20-50 \mathrm{meV} \mathrm{THz}$ band. This may also explain why the intersubband spectral features in many of the reports of $\mathrm{THz}$ electroluminescence are so broad and centered at similar energies despite fairly different active region designs. ${ }^{22,23}$

On the plus side however, these properties suggest that SiGe:P might be a practical route toward a wavelength tun- 
able silicon impurity laser. The potential gain peak for $\mathrm{SiGe}: \mathrm{P}$ is much broader than the narrow donor lines in $\mathrm{Si}: \mathrm{P}$ but the long lifetime suggests that a population inversion is still achievable. It is likely, however, that higher doping densities will be needed in SiGe to achieve similar peak gain values to that found in pure Si:P in order to overcome the cavity losses and reach the lasing threshold. Following a similar logic, the highly broadened nature of the donor lines means that $\mathrm{SiGe:P}$ is potentially a good material to realize fast sensitive $\mathrm{THz}$ detectors with a broader spectral bandwidth.

The possibility of realizing a qubit using single phosphorus donors in silicon has also generated considerable interest. While many quantum-computing proposals make use of the spin properties of the donor, some schemes such as that proposed by Stoneham et al. ${ }^{24}$ make use of the farinfrared energy levels. Indeed, there have been significant experimental developments in this area recently. 5 ,6 Stoneham's theory depends crucially on the donor states having a spread of energies while still retaining a sensible lifetime. Our paper shows that it would be possible to engineer the electronic energy level structure of an isolated phosphorus donor by altering the number of $\mathrm{Ge}$ atoms in its vicinity without detrimentally affecting the excited electronic state lifetimes.

\section{CONCLUSIONS}

FT-IR spectroscopy of SiGe single crystals shows that a relatively small content of $\mathrm{Ge}$ in $\mathrm{Si}_{1-x} \mathrm{Ge}_{x}$ causes a large broadening of the phosphorus donor lines. Direct lifetime measurements, however, show that while the lifetime is shorter than in pure $\mathrm{Si}: \mathrm{P}\left(118 \pm 14\right.$ ps for the broadened $2 p_{ \pm}$ peak in SiGe:P, as compared to $158 \pm 12$ ps for the $2 p_{ \pm}$line in pure $\mathrm{Si}: \mathrm{P}),{ }^{5}$ it has not collapsed to $<1$ ps (calculated by applying the Heisenberg uncertainty principle to the farinfrared absorbance spectrum). Furthermore, the observed change in lifetime with temperature is not reflected in the FT-IR absorbance spectrum. This experimental result provides direct evidence that the line broadening is inhomogenous and our simple quantitative line broadening model shows that it is sufficient to take into account the local variation in permittivity due to the random nature of the binary alloy in order to fully describe the broadening. The model provides additional compelling evidence that the broadening is inhomogenous.

\section{ACKNOWLEDGMENTS}

We acknowledge financial support from the UK Engineering and Physical Sciences Research Council (Grants No. EP/ E061265/1 and EP/H026622) and the Netherlands Organisation for Scientific Research. We thank the British Council and the Deutscher Akademischer Austausch Dienst for supporting this collaboration through ARC under Grant No. 1361 and DAAD under Project No. 50023075. *stephen.lynch@ucl.ac.uk

${ }^{\dagger}$ German Aerospace Center (DLR), Institute of Planetary Research, Rutherfordstr. 2, 12489 Berlin, Germany.

${ }^{1}$ B. E. Kane, Nature (London) 393, 133 (1998).

${ }^{2}$ A. M. Tyryshkin, S. A. Lyon, A. V. Astashkin, and A. M. Raitsimring, Phys. Rev. B 68, 193207 (2003).

${ }^{3}$ G. W. Morley, D. R. McCamey, H. A. Seipel, L. C. Brunel, J. van Tol, and C. Boehme, Phys. Rev. Lett. 101, 207602 (2008).

${ }^{4}$ D. Karaiskaj, J. A. H. Stotz, T. Meyer, M. L. W. Thewalt, and M. Cardona, Phys. Rev. Lett. 90, 186402 (2003).

${ }^{5}$ N. Q. Vinh, P. T. Greenland, K. Litvinenko, B. Redlich, A. F. G. van der Meer, S. A. Lynch, M. Warner, A. M. Stoneham, G. Aeppli, D. J. Paul, C. R. Pidgeon, and B. N. Murdin, Proc. Natl. Acad. Sci. U.S.A. 105, 10649 (2008).

${ }^{6}$ P. T. Greenland, S. A. Lynch, A. F. G. van der Meer, B. N. Murdin, C. R. Pidgeon, B. Redlich, N. Q. Vinh, and G. Aeppli, Nature (London) 465, 1057 (2010).

${ }^{7}$ S. G. Pavlov, R. Kh. Zhukavin, E. E. Orlova, V. N. Shastin, A. V. Kirsanov, H.-W. Hübers, K. Auen, and H. Riemann, Phys. Rev. Lett. 84, 5220 (2000).

${ }^{8}$ S. G. Pavlov, H.-W. Hübers, M. H. Rümmeli, R. Kh. Zhukavin, E. E. Orlova, V. N. Shastin, and H. Riemann, Appl. Phys. Lett. 80, 4717 (2002).

${ }^{9}$ H.-W. Hübers, S. G. Pavlov, H. Riemann, N. V. Abrosimov, R. Kh. Zhukavin, and V. N. Shastin, Appl. Phys. Lett. 84, 3600 (2004).

${ }^{10}$ S. G. Pavlov, H.-W. Hübers, J. N. Hovenier, T. O. Klaassen, D.
A. Carder, P. J. Phillips, B. Redlich, H. Riemann, R. Kh. Zhukavin, and V. N. Shastin, Phys. Rev. Lett. 96, 037404 (2006).

${ }^{11}$ T. Ghani, M. Armstrong, C. Anuth, M. Bost, P. Charvat, T. Hoffmann, K. Johnson, C. Kenyon, J. Klaus, B. McIntyre, K. Mistry, A. Murthy, J. Stanford, M. Silberstein, S. Sivakumar, P. Smith, K. Zawadzki, S. Thompson, and M. Bohr, Tech. Dig. - Int. Electron Devices Meet. 2003, 11.6.1.

${ }^{12}$ N. V. Abrosimov, S. N. Rossolenko, V. Alex, A. Gerhardt, and W. Schröder, J. Cryst. Growth 166, 657 (1996).

${ }^{13}$ S. A. Lynch, P. Townsend, G. Matmon, D. J. Paul, M. Bain, H. S. Gamble, J. Zhang, Z. Ikonic, R. W. Kelsall, and P. Harrison, Appl. Phys. Lett. 87, 101114 (2005).

${ }^{14}$ M. Franz, K. Pressel, A. Barz, P. Dold, and K. W. Benz, J. Vac. Sci. Technol. B 16, 1717 (1998).

${ }^{15}$ E. F. Schubert, E. O. Göbel, Y. Horikoshi, K. Ploog, and H. J. Queisser, Phys. Rev. B 30, 813 (1984).

${ }^{16} \mathrm{P}$. Yu and M. Cardona, Fundamentals of Semiconductors, Advanced Texts in Physics, 3rd ed. (Springer, New York, 2001).

${ }^{17}$ J. P. Dismukes, L. Ekstrom, and R. J. Paff, J. Phys. Chem. 68, 3021 (1964).

${ }^{18}$ J. Humlíček, A. Röseler, T. Zettler, M. G. Kekoua, and E. V. Khoutsishvili, Appl. Opt. 31, 90 (1992).

${ }^{19}$ R. Krüssmann, H. Vollmer, and R. Labusch, Phys. Status Solidi B 118, 275 (1983).

${ }^{20}$ S. A. Lynch, D. J. Paul, P. Townsend, G. Matmon, Z. Suet, R. W. Kelsall, Z. Ikonic, P. Harrison, J. Zhang, D. J. Norris, A. G. 
Cullis, C. R. Pidgeon, P. Murzyn, B. N. Murdin, M. Bain, H. S. Gamble, M. Zhao, and W.-X. Ni, IEEE J. Sel. Top. Quantum Electron. 12, 1570 (2006).

${ }^{21}$ G. Matmon, D. J. Paul, L. Lever, M. Califano, Z. Ikonic, R. W. Kelsall, J. Zhang, D. Chrastina, G. Isella, H. von Känel, E. Müller, and A. Neels, J. Appl. Phys. 107, 053109 (2010).

${ }^{22}$ S. A. Lynch, R. Bates, D. J. Paul, D. J. Norris, A. G. Cullis, Z.
Ikonic, R. W. Kelsall, P. Harrison, D. D. Arnone, and C. R. Pidgeon, Appl. Phys. Lett. 81, 1543 (2002).

${ }^{23}$ R. Bates, S. A. Lynch, D. J. Paul, Z. Ikonic, R. W. Kelsall, P. Harrison, S. L. Liew, D. J. Norris, A. G. Cullis, W. R. Tribe, and D. D. Arnone, Appl. Phys. Lett. 83, 4092 (2003).

${ }^{24}$ A. M. Stoneham, A. J. Fisher, and P. T. Greenland, J. Phys.: Condens. Matter 15, L447 (2003). 